\title{
La aloinmunoterapia en aborto habitual. Una controversia abierta*
}

\author{
Angela P. Cadavid, MD, MS*
}

\begin{abstract}
RESUMEN: Se presenta una revisión crítica de los resultados del Meta-análisis del Estudio Colaborativo Internacional sobre Inmunoterapia en Aborto habitual y se discuten los principales puntos de controversia, a la luz del VI Congreso Internacional de Inmunología de la Reproducción, en Washington, julio de 1995.
\end{abstract}

PALABRAS CLAVES: Aborto recurrente espontáneo, inmunoterapia, transfusiones, inmunología de la gestación.

SUMMARY: The article presents a critical review of the results of a Worldwide Collaborative Observational Study and Meta-analysis on allogeneic leukocyte immunotherapy for recurrent spontaneous abortion. The main controversial aspects of that study are discussed to the light of the results presented at the VI International Congress of Reproduction Immunology, held in Washington in july 1995.

KEY WORDS: Recurrent spontaneous abortion, immunotherapy, transfusions, immunology of gestation.

\section{Introducción}

El aborto recurrente espontáneo (ARE), es una complicación del embarazo que afecta aproximadamente un 2-3\% de las parejas y aunque algunas causas se han identificado (endocrinas, cromosómicas, anatómicas, infecciosas, inmunológicas, etc.), todavía persiste un $60 \%$ de abortos con causa desconocida.

Con el descubrimiento de los antígenos de histocompatibilidad, surgió la pregunta central de la inmunología de reproducción de por qué la madre no rechaza su feto semialogénico. Hoy en día, está ampliamente aceptado que en la gestación normal la madre responde contra el feto, pero existen diferentes hipótesis sobre la forma cómo esta respuesta es modulada (1-5). Fue así como se empezó a considerar que una alteración en esta modulación podía ser una causa inmunológica del ARE y esta fue la primera patología de la gestación que se asoció con el sistema inmune. Posteriormente, se postuló que otras alteraciones de la reproducción podían tener compromiso inmunológico.

La terapia con leucocitos fue a su vez, la primera intervención inmunológica del aborto (6). Se han utilizado otros tratamientos inmunológicos como la infusión con membranas trofoblásticas, la inmunoglobulina intravenosa, los intralípidos, el adyuvante de Freund y algunas citoquinas. Otros tratamientos no inmunológicos como la sicoterapia, el cerclaje y la gonadotrofina coriónica humana, se han descrito como efectivos.

Con este artículo se pretende: primero, revisar y comentar los hallazgos del Meta-análisis del Estudio Colaborativo Internacional de la Aloinmunoterapia en ARE; segundo, discutir los principales puntos de contro-

* Programa Reproducción, Facultad de Medicina, Universidad de Antioquia, A.A. 1226, Medellín-Colombia. versia a la luz del VI Congreso Internacional de Inmunología de la Reproducción realizado en Washington en julio de 1995.

Para efectos de este artículo, se llamará inmunoterapia a la inyección parenteral de leucocitos alogénicos. No existe un protocolo unificado de inmunoterapia: unos grupos utilizan como donante de la sangre el esposo, algunos un tercero y otros una mezcla; unos aplican leucocitos totales y otros mononucleares; la vía puede ser intravenosa, subcutánea, intradérmica o combinaciones. El número de células es también variable, al igual que el tiempo y la frecuencia de las inmunizaciones (preconcepción solamente, preconcepción con reinmunizaciones durante el embarazo o iniciación de la terapia cuando la gestación se ha diagnosticado).

El uso de las transfusiones sanguíneas como terapia inmunológica, no es innovador. Ellas tuvieron su aplicación clínica en la década de los 70 cuando se observó que los pacientes que habían sido transfundidos en alguna ocasión, tenían un mejor pronóstico en la sobrevida de los trasplantes (7); se empezaron a utilizar entonces, en la modalidad de transfusiones específicas y transfusiones al azar. Años más tarde, la influencia de las transfusiones en la supervivencia de los injertos disminuyó, posiblemente por el uso de la ciclosporina como droga inmunosupresora.

La inmunoterapia está basada en evidencias clínicas (8-10) y experimentales (11-12) que demuestran que la respuesta alogénica, en la gestación normal y la inducida por las inmunizaciones, no sólo no es dañina para el feto, sino que por el contrario es protectora y además favorece el crecimiento y desarrollo del feto y de la placenta. Algunas de estas observaciones se han tratado de sincretizar en la hipótesis inmunotrópica (13).

Los resultados de la inmunoterapia son muy controvertidos. Existen estudios que respaldan su efectividad $(6,14)$, pero otros que no encuentran diferencia significativa entre el grupo control y el tratado (15). La expli- 
cación que se ha dado es que los estudios se han hecho con muestras pequeñas, con poblaciones heterogéneas que tienen diferentes factores pronósticos y además, puede haber interferencia por la cointervención en el grupo control, ya que los placebos utilizados pueden tener efecto.

\section{Diseño del meta-análisis}

Para tratar de resolver la controversia, el Comité de Etica de la Sociedad Americana de Inmunología de la Reproducción organizó un Estudio Colaborativo Internacional en el cual convocó a los grupos que utilizaran inmunoterapia con leucocitos para el tratamiento del ARE y que tuvieran además grupos control sin tratamiento.

Quince centros de diferentes partes del mundo cumplieron con los criterios y llenaron un formato con 140 variables con los datos de las pacientes. De estos estudios, siete eran randomizados y doble ciego, dos randomizados pero no ciegos y seis eran estudios comparativos en los cuales el tratamiento se hizo por preferencia y no al azar (16).

El principal objetivo del metanálisis era resolver la pregunta de si la inmunoterapia mejora el porcentaje de nacidos vivos. Adicionalmente se podrían identificar los factores pronósticos asociados con la probabilidad de un hijo vivo en parejas con ARE (16).

Los datos fueron analizados por dos equipos diferentes que seleccionaron las pacientes de acuerdo con los siguientes criterios: pacientes con tres o más abortos, no más de un hijo vivo con cualquier pareja, no evidencia de otras causas de aborto incluyendo los autoinmune, no cointervención simultánea durante el embarazo con otras drogas como aspirina, heparina, corticosteroides o progesterona, en el análisis No. 1; pacientes con tres o más embarazos intrauterinos de menos de 20 semanas y no más de un hijo vivo con la pareja actual en el análisis No. 2 (16).

Los factores pronósticos considerados por ambos grupos fueron la edad materna y la frecuencia de HLA compartidos. En el análisis No. 1 estudiaron además número de abortos previos, cantidad de leucocitos administrados y número de tratamientos recibidos, tiempo entre la inmunización y la concepción y nacidos vivos previos; el análisis No. 2 incluyó por su parte presencia de autoinmunidad (anticuerpos antinucleares o anticardiolipina positivos), desórdenes de coagulación, alteraciones menstruales, historia de infertilidad, anormalidades tubáricas, defectos uterinos y tratamiento con células autólogas (16).

Cada uno de los grupos de análisis utilizó diferentes pruebas estadísticas, pero en términos generales determinaron el riesgo relativo (RR) para expresar la probabilidad de un hijo vivo; el test de Mantel-Haenszel para comparar el porcentaje de nacidos vivos entre los grupos; el test de Kruskall-Wallis, el test exacto de Fischer y el chi-cuadrado (en un grupo) y la regresión logística (en el otro grupo) para estudiar la influencia de las variables pronósticas; y la estadística de Breslow-Day para probar la homogeneidad entre los centros (16).

Para efecto del análisis se consideró el tratamiento con linfocitos paternos o de donante como un solo grupo, porque no hubo diferencia significativa entre ellos, y cada grupo por sí mismo era muy pequeño para un análisis independiente con suficiente poder estadístico. Lo mismo se hizo en los controles con los grupos de notratamiento, tratamiento con linfocitos autólogos o solución salina (16).

Se consideró como falla todos los resultados diferentes a un hijo vivo (continuación de la infertilidad, ectópico, aborto espontáneo o terapéutico, etc).

\section{Resultados del meta-análisis}

Los resultados de los dos grupos de análisis fueron muy similares, como se muestra a continuación:

$\begin{array}{lcr}\text { Grupo de Análisis } & \text { No. } 1 & \text { No. } 2 \\ \text { Pacientes inmunizadas } & 231 & 240 \\ \text { Pacientes control } & 199 & 209 \\ \text { Efecto relativo del tratamiento } & 1.16 & 1.21 \\ \text { Nacidos vivos/100 pacientes tratadas } & 8 & 10 \\ \text { Beneficio } & 1: 13 & 1: 10\end{array}$

Las pacientes abortadoras primarias tuvieron un porcentaje de éxito mayor con la inmunoterapia que las abortadoras secundarias, en las cuales no se observó diferencia con el grupo control. La probabilidad de nacidos vivos fue mayor en las mujeres que no tenían anticuerpos antes del tratamiento pero demostraron seroconversión comparado con las que no sero-convirtieron o tenían anticuerpos antes del tratamiento. La autoinmunidad parece ser un factor pronóstico negativo para el efecto de la inmunoterapia (62\% más bajo) (41).

La administración intravenosa de cualquiera de los tratamientos, se asoció con un $21 \%$ de disminución en la probabilidad de un hijo vivo. La edad materna tuvo relación en el análisis No. 1, pero no en el No. 2 ; no se encontró relación con la frecuencia de HLA compartidos, número de células administradas, tiempo transcurrido entre la inmunización y el embarazo (16).

Se reportaron más efectos colaterales $(1.6 \%)$ en el grupo de inmunoterapia que en el control, pero el chance de beneficio del tratamiento fue 5 a 6 veces más grande que el riesgo de un efecto colateral. Estos efectos incluyeron hepatitis, CMV, resfriados, fiebres, anticuerpos antieritrocitos o antiplaquetas. Algunas pacientes desarrollaron características clínicas sugestivas de autoinmunidad como exantema, artritis, trombosis y fenómeno de Raynaud; pero no hubo diferencia significativa entre el grupo de terapia y el control. Además las anormalidades autoinmunes no habían sido rigurosamente descartadas antes de entrar al estudio. Las complicaciones de los recién nacidos fueron similares en ambos grupos, pero no hay estudios formales de seguimiento a largo plazo (16).

Daya y Gunby, por su parte, analizaron los datos de un grupo más seleccionado de pacientes que incluía solamente abortadoras primarias, con tres o más abortos, sin ninguna otra causa identificable de aborto y sin cointervención simultánea. El objetivo era determinar si con criterios más estrictos de selección, con los cuales otras causas de aborto se hubieran descartado, se altera- 
ban los resultados de la efectividad de la inmunoterapia por disminuir la probabilidad de cura espontánea en el grupo control (16).

Para expresar la eficacia de la terapia, calcularon el efecto absoluto del tratamiento y el número necesario de pacientes a tratar para obtener como resultado un hijo vivo, NNT, porque esta estadística incorpora el riesgo basal en las pacientes no tratadas y provee un estimado del esfuerzo y costo asociado con la terapia. En este análisis se incluyeron 285 pacientes (150 inmunoterapia y 135 controles). Los resultados indicaron que la inmunoterapia significativamente mejoró la probabilidad de nacidos vivos $(R R=1.46)$, el porcentaje de nacidos vivos fue de $60.7 \%$ comparado con $44.4 \%$ en el grupo control $(\mathrm{P}=0.006)$, el efecto absoluto del tratamiento fue de $16.3 \%$, el NNT fue 6 (comparado con un NNT de 11 en el grupo completo) (17).

En cuanto a las variables pronósticas, sólo encontraron correlación negativa entre el número de abortos previos y el porcentaje de nacidos vivos. La inmunoterapia al parecer contrarresta este efecto negativo en la probabilidad de nacidos vivos. Las otras variables no afectaron los resultados (17).

Las pacientes que no se embarazaron en el período de observación eran consideradas como falla del tratamiento. Los autores repitieron el análisis después de excluir estas pacientes (14 del grupo tratado y 16 en el control), y los resultados fueron similares. Además se puede inferir que la inmunoterapia no parece estar asociada con posterior reducción o aumento en la fertilidad (17).

\section{Discusión}

$\mathrm{Si}$ se revisan las publicaciones previas de factores pronósticos en los resultados de la inmunoterapia, se pueden encontrar algunas controversias. Con relación a infertilidad y ARE, Coulam y cols mostraron una mayor frecuencia de aborto espontáneo entre parejas infértiles y una mayor prevalencia de infertilidad entre pacientes con ARE, comparado con población normal. Sugi y cols habían sugerido un efecto benéfico de las inmunizaciones en la infertilidad por una disminución de los anticuerpos anti-espermatozoides después de la inmunoterapia. En el Centro de Tratamiento de la Esterilidad en Montpellier, no encontraron beneficio de la inmunoterapia en los grupos de infertilidad inexplicada o en los de falla de implantación después de FIV. Cowchock y cols, en un estudio prospectivo comparando un grupo de pacientes inmunizadas antes de la concepción con otro inmunizado seis semanas después de la última menstruación, encontraron que las mujeres con ARE no tenían un alto riesgo de infertilidad secundaria y que la inmunización preembarazo no afectaba la proporción de embarazos en estas pacientes. Se excluyeron del estudio las pacientes con historia previa de alteraciones en la fecundación o pérdidas repetidas tempranas.

Otras variables como número de células administradas también parecía tener alguna influencia. Smith y cols, reportaron que cantidades menores de 150 millones eran insuficientes y cantidades mayores de 500 millones, podían tener un efecto negativo. Aoki y cols, describie- ron efectos significativamente mayores en pacientes inmunizadas con 100-200 millones de células que en las inmunizadas con 1 millón.

En cuanto al tiempo transcurrido entre la inmunización y la gestación, Mowbray y cols, reportaron que el resultado era igualmente efectivo si la gestación ocurría en los primeros 40 días; Aoki encontró una mayor efectividad si el tratamiento se iniciaba en las primeras cuatro semanas de la gestación (49). Reginald, administró exitosamente un esquema de inmunización inicial y una reinmunización en las primeras semanas de la gestación.

Con relación a la aparición de autoinmunidad después de la terapia con linfocitos, Moncayo y cols, en un estudio retrospectivo, encontraron un incremento de los niveles de IgG e IgM contra cardiolipina en pacientes tratadas con inmunoterapia. Por su parte Christiansen y cols, en un estudio prospectivo, no encontraron cambios en los niveles de anticuerpos anticardiolipina y además no encontraron correlación positiva entre la presencia de estos anticuerpos y el riesgo de aborto en estas pacientes.

En general, el porcentaje bajo de éxito de la inmunoterapia en el Estudio Colaborativo, pudiera indicar que la inmunoterapia sólo tiene efecto benéfico en un pequeño grupo de la población afectada por el trastorno inmunológico o, bien pudiera ser una indicación de que la inmunoterapia es altamente efectiva, pero el defecto inmunológico no se está diagnosticando apropiadamente. Si lo primero es correcto se deben buscar mejores terapias; si lo correcto es lo segundo, se debe mejorar el diagnóstico para seleccionar las pacientes que se puedan beneficiar del tratamiento (17): De todas maneras se requiere profundizar en los aspectos básicos del problema hasta llegar a conocer los mecanismos patogénicos para podernos acercar a los mecanismos de la inmunoterapia, o viceversa.

Para interpretar la eficacia de la inmunoterapia, es necesario hacer otros cálculos adicionales relacionados con la etiología genética. Estudios recientes en muestras de vellosidades coriónicas de una nueva pérdida, en mujeres con historia de aborto habitual, mostraron $60 \%$ de anormalidades cromosómicas (18) y aunque pueden representar mosaicos sin anormalidades fetales, tampoco las descarta como causa de aborto. Si se tiene en cuenta que la inmunoterapia sólo actuaría en el $40 \%$ restante, los cálculos de efectividad serían mayores que un $8-10 \%$, para rescatar lo rescatable (19).

Hasta el momento no hay un criterio universalmente aceptado para la evaluación de ARE, llevando a una gran variabilidad entre los centros. Aunque todos realizan unas pruebas básicas, algunos tienen unos criterios de exclusión más estrictos que otros. Se podría especular que entre más riguroso el criterio de exclusión se puede observar un efecto del tratamiento más alto, porque el porcentaje de cura espontáneo en el grupo control será más bajo (17).

Cowchock y cols, reportaron que los métodos inmunológicos de diagnóstico utilizados hasta ahora no tienen un valor predictivo en el resultado del embarazo, independiente de que tengan o no tengan tratamiento (20). Algunos estudios no han encontrado correlación de anticuerpos antipaternos con el éxito; otros postulan que 
los anticuerpos pre-existentes predicen el éxito o que el bloqueo pre-existente predice la falla. Mowbray reportó que una respuesta de anticuerpo se correlaciona con la duración de la protección; en la ausencia de anticuerpos la rata de éxito cae a $30-35 \%$ después de 12 semanas y puede ser restaurada con una reinmunización. Smith y Cowchock informaron una disminución de la protección después de 12 semanas.

La falta de métodos diagnósticos adecuados, es en parte un reflejo de los vacíos que existen en el entendimiento de la relación materno-fetal. La gestación en los placentados puede ser mirada en el contexto de los transplantes, donde el embrión es un aloinjerto y consecuentemente puede estimular una respuesta de rechazo específica; o se puede considerar en el contexto de los tumores y, entonces, otros mecanismos de rechazo noespecíficos de antígeno, como las células NK y los macrófagos podrían estar jugando el rol mayor (22).

Esta diferencia es importante tanto para el diagnóstico como para el tratamiento. Si el aborto se da por una reacción de rechazo o por una reacción de injerto versus huésped, entonces la administración pasiva o la inducción de la producción de anticuerpos que bloqueen algún antígeno relevante o que bloqueen el receptor de las células $\mathrm{T}$ alo-reactivas serían el tratamiento de elección. En este sentido el suero de mujeres multíparas sería benéfico. Si el aborto es por activación de las células NK, se pudiera bloquear esta activación con estímulos noespecíficos de antígeno como citoquinas, intralípidos, adyuvantes, drogas inmunoestimuladoras o, como más recientemente se ha sugerido con inmunoglobulina intravenosa.

Es importante hacer énfasis en las células con morfología NK en la decidua: los LGL en humanos y las GMG en ratones, constituyen la población más frecuente de leucocitos en decidua de primer trimestre y prácticamente desaparecen en placenta a término. Su función no está muy clara, pero pueden estar comprometidas en controlar la invasión del trofoblasto; cuando son estimuladas con IL-2, se convierten en células LAK con actividad lítica contra trofoblasto. Se ha sugerido que su activación es un factor causal muy importante en el aborto. Además de su actividad lítica, pudieran estar ejerciendo alguna función en la producción de citoquinas. Croy y cols, estudiando placentas de ratones deficientes en células $\mathrm{NK}$, encontraron que tenían placentas de menor tamaño comparada con las de ratones normales. Esto la llevó a sugerir que las células NK, y no las células T, podrían ser las responsables de la producción de las citoquinas inmunotrópicas en la interfase materno-fetal (21).

La investigación en el campo de la inmunología de la reproducción es muy prolífica y cuando se revisa la literatura, se encuentra que cada día los diferentes grupos de trabajo están proponiendo nuevas moléculas y mecanismos como protagonistas de esta compleja interacción materno-fetal. Es así como Kovats y cols, han propuesto que la expresión del HLA-G puede proteger el trofoblasto de la lisis por células NK y estimular la producción de algunas citoquinas supresoras y de otras inmunotrópicas. Nowbray y cols, están trabajando en el antígeno R80K presente en trofoblasto de placentas a término; este antígeno es muy polimórfico y parece estar determinado por los genes paternos e induce una respuesta de anticuerpos por parte de la madre. Un anticuerpo monoclonal dirigido contra este antígeno bloquea la lisis de células NK.

Por su parte, Clark y cols, han centrado su investigación en una molécula supresora relacionada con el TGFb, que es producida por células deciduales CD56+ y se encuentra disminuida en los casos de aborto, con un aumento del TNF-a (3). Beaman y cols, consideran que una proteína codificada por el gen TJ6, que es supresora de las células $\mathrm{T}$ efectoras, podría ser la responsable de la tolerancia materna ya que está involucrada en la deleción clonal de la apoptosis inducida por dexametasona en timocitos; la han encontrado expresada en linfocitos B de mujeres gestantes normales y en las células NK deciduales en mujeres que sufren aborto. Los grupos de Wegmann (4) y Chaouat, han encontrado que citoquinas como la IL10, el GM-CSF y la IL-3, pueden tener funciones supresoras. Ossa y cols, en nuestro grupo de trabajo, hemos propuesto que el aborto es un tipo de reacción de injerto versus huésped donde las células de la madre que alcanzan el feto, son el injerto y el feto huésped, y que la falta de una respuesta anti-idiopática materna que controle la reacción de injerto vs huésped, sería la causa de la pérdida fetal (5).

El mecanismo de acción de la inmunoterapia no se conoce, pero la mayoría de los investigadores han tratado de estudiar posibles mecanismos en su respectivo modelo. Se ha reportado aumento de factores bloqueadores (10), anticuerpos anti-idiotípicos (22), disminución de células citotóxicas (22), incremento de receptores de progesterona en células CD8+ (23), bloqueo de actividad NK (24), disminución de TNF-a, aumento de TGF-b (3), aumento de citoquinas anti-inflamatorias como IL-4 e IL-10. En este mismo sentido, el mecanismo de acción de las transfusiones sanguíneas como terapia no se aclaró, pero se han asociado con un efecto inmunosupresor no caracterizado que lleva a tolerancia a transplantes, aumento de susceptibilidad a infecciones virales o bacterianas y a un rompimiento de la vigilancia inmune contra tumores. Más recientemente se ha postulado que el microquimerismo celular (persistencia de células mononucleares del donante en el receptor), podría ser uno de los mecanismos de tolerancia o anergia del receptor. Sería importante demostrar que el quimerismo tiene un papel en la inmunoterapia y que posiblemente, el efecto positivo de la misma sea dependiente de esta variable.

Un trabajo reciente realizado por Croy y cols, demuestra de una manera muy elegante y contundente, el quimerismo de células maternas en fetos de ratones, por el tráfico de células a través de la placenta. Utilizaron tanto embriones normales como scid (deficientes en células T y B) y los transfirieron a madres seudopreñadas que eran transgénicas para el gen Lac, lo cual permitió detectar las células maternas en los fetos por la detección del producto de este gen, que es la b-galactosidasa y que le da un color azul a las células. Todavía no se ha identificado el fenotipo de estas células ni su función. 
Para nuestro grupo, este trabajo se constituye en un pilar fundamental para nuestra hipótesis porque primero, se demuestra el tráfico de células a través de la placenta y segundo, el establecimiento de quimerismo en el feto. Queda por estudiar qué papel está jugando este quimerismo en la tolerancia materna y los mecanismos que controlan la reacción de injerto vs huésped.

En resumen, la eficacia de la terapia con leucocitos alogénicos no pudo ser confirmada ni descartada con el Estudio Colaborativo Internacional. Lo que sí parece ser un consenso, es que se necesitan métodos diagnósticos inmunológicos más adecuados para seleccionar las pacientes que se pudieran beneficiar de la inmunoterapia. Una solución adicional, sería el uso de terapias inmunológicas alternativas como la inmunoglobulina intravenosa; su mecanismo de acción no está bien esclarecido, pero además de su efecto pasivo por la administración de gran variedad y cantidad de anticuerpos, se considera que tiene otros efectos inmunomodulatorios por bloqueo de receptores $\mathrm{Fc}$, reducción de la actividad NK, bloqueo de receptores del complemento (25); además podría estar actuando en la restauración de la red idiotípica.

Desafortunadamente su uso es muy limitado en nuestro medio por los altos costos que conlleva.

En la práctica clínica, es muy importante estudiar y tratar las causas más comunes de aborto y no considerar la inmunoterapia como un "refuerzo" o un reemplazo de los otros tratamientos. Además se debe garantizar que las parejas reciban toda la información pertinente sobre los riesgos, los beneficios y las incertidumbres que existan alrededor de la inmunoterapia, para que ellos, de una manera consciente tomen la decisión.

\section{Agradecimientos}

A COLCIENCIAS por la financiación a través del proyecto 1115-070491 y del Programa de Formación de Recursos Humanos.

\section{BIBLIOGRAFIA}

1. Bell SC., Billington D. Anti-fetal allo-antibody in the pregnant female. Immunol Rev 1983; 75: 5-30.

2. Joseph S., Butler L., Liao SK., Singal P. Antiidiotypic antibodies associated with successful pregnancy. Transplant Proc 1985; 17(1): 909-910.

3. Clark DA., Lea RG., Flanders KC., Banwatt D., Chaouat G. Role of unique species of TGF-b in preventing rejection of the conceptus during pregnancy. In: Gergely J., Benczúr M., Erdei N., et al., eds. Progress in Immunology VIII. Springer Verlag Budapest; 1986; 841 847.

4. Wegmann TG., Lin H., Guilbert L., Mossman TR. Bidirectional cytokine interactions in the maternal-fetal relationship: is successful pregnancy a $\mathrm{TH}_{2}$ phenomenon? Immunol Today 1993; 353-356.

5. Ossa JE., Cadavid AP., Maldonado JG. Is the immune system necessary for placental reproduction? A hypothesis on the mechanisms of alloimmunotherapy in recurrent spontaneous abortion. Med Hypoth 1994; 42: 193-197.

6. Mowbray JF., Underwood JL., Michel M., Forbes PB., Beard RW. Immunisation with paternal lymphocytes in women with recurrent miscarriage. Lancet 1987; 2: 679-680.

7. Opelz G., Terasaki PI. Prolongation effect of blood transfusions on kidney graft survival 1976; 22(4): 380-383.

8. Beer AE., Semprini AE., Xiaoyu Z., Quebbeman JF. Pregnancy outcome in human couples with recurrent spontaneous abortions: HLA antigen profiles; HLA antigen sharing; female serum MLR blocking factors; and paternal leukocyte immunization. Expl Clin Immunogenet 1985; 2: 137-153.

9. Mowbray JF., Underwood JL. Effect of paternal lymphocyte immunisation on birth-weight and pregnancy outcome. In: Chaouat G., Mowbray J., eds. Cellular and Molecular Biology of the MaternoFetal Relationship. Collóque INSERM? John Libbey Eurotext Ltd, 1991; 212: 295-302.

10. Takakuwa K., Kanazawa K., Takeuchi S. Production of blocking antibodies by vaccination with husband's lymphocytes in unexplained recurrent aborters: the role in successful pregnancy. Am J Reprod Immunol Microbiol 1986; 10: 1-9.

11. Billington WD. Influence of immunological dissimilarity of mother and foetus on size of placenta in mouse. Nature (Lond.) 1964; 202: 317-318.

12. Chaouat G., Kolb JP., Kiger N., Stanislawski M., Wegmann TG. Immunological consequences of vaccination against abortion in mice. J Immunol 1985; 134(3): 1594-1598.

13. Wegmann TG. Maternal T cells promote placental growth and prevent spontaneous abortion. Immunol Letters 1988; 17: 297-302.
14. Beer AE., KwakJY. What is the evidence for immunological pregnancy loss? lymphocyte immunization the supportive view. In: Chaouat G., Mowbray J., eds. Cellular and Molecular Biology of the MaternoFetal Relationship. Colloque INSERM/John Libbey Eurotext Ltd, 1991.

15. Cauchi MN., Lim D., Young DE., Loss M., Pepperell RJ. Treatment of recurrent aborters by immunizations with paternal cells-controlled trial. Am J Reprod Immunol 1991; 25: 16-17.

16. The Recurrent Miscarriage Immunotherapy Trialists Group. Worldwide collaborative observational study and meta-analysis on allogenic leukocyte immunotherapy for recurrent spontaneous abortion. Am J Reprod Immunol 1994; 32: 55-72.

17. Daya S., Gunby J and The Recurrent Miscarriage Immunotherapy Trialist Group. The effectiveness of allogeneic leukocyte immunization in unexplained primary recurrent spontaneous abortion. Am J Reprod Immunol 1994; 32: 294-302.

18. Stern JJ., Dorfmann A., Gutiérrez-Najar AJ., Cerillo M., Coulam CB Frequency of abnormal karyotypes among abortuses from women with and without a history of recurrent spontaneous abortion. Fertil Steril (Suppl) 1995, (in press).

19. Clark DA., Daya S., Coulam CB., Gunby J. The modern preventative treatment of recurrent miscarriage. Br. J. Obstet. Gynecol. 1995 (in press).

20. Cowchock FS., Smith JB. Predictors for liver birth after unexplained spontaneous abortions: correlations between immunologic test results, obstetrics histories, and outcome of next pregnancy without treatment. Am. J. Obstet. Gynecol. 1992; 167: 1208-1212.

21. Croy BA. Studies of pregnancy associated granulated metrial gland cells in mutant and transgenic mice. Oral presentation Sixth Internacional Congress. Am J Reprod Immunol 1995; 33: 431.

22. Toshitaka S., Tsunehisa M., Tetsuo M. A possible mechanism of immunotherapy for patients with recurrent spontaneous abortion. Am J Reprod Immunol 1991; 25: 185-189.

23. Szekeres-Bartho J., Varga P., Kapovic M., Chaouat G. Progesterone dependent immunomodulation during pregnancy. In: Chaouat G., Mowbray J eds. Cellular and Molecular Biology of the Materno-Fetal Relationship. Colloque INSERM? John Libbey Eurotext Ltd, 1991; 212: 309-316.

24. Higuchi K., Aoki K., Kimbara T et al. Suppression of antural killer cell activity by monocytes following immunotherapy for recurrent spontaneous abortion. Am J Reprod Immunol 1995; 33: 221-227.

25. Newlan AC. The use and mechanisms of action of intravenous immunoglobulin: An update. Br J Hematol 1989; 72(3): 301-305. 